\title{
Jutta Wagner
}

\author{
Rechtsanwältin und Notarin, Präsidentin des djb von 2005 bis 2011 \\ Juristinnen machen Karriere - wir stellen sie vor
}

Das Interview führte Anke Gimbal, Bundesgeschäftsführerin des djb, im Oktober 2011 in Berlin.

\section{Warum haben Sie Jura studiert?}

Ich habe Jura studiert, weil ich Journalistin werden wollte. Mir hatte ein hoher Springer-Chef geraten, ich sollte alles studieren, wenn ich Journalistin werden will, bloß nicht Publizistik. Dem bin ich gefolgt. Ich dachte, Jura hat Hand und Fuß. Man lernt etwas Nützliches fürs Leben und man lernt den Umgang mit Sprache.

\section{War dies die richtige Entscheidung?}

Ja, es war die richtige Entscheidung, auch wenn ich nun keine Journalistin bin. Aber mein Beruf macht mir Spaß.

\section{War Ihr Ziel, Anwältin zu werden, oder hat sich das „so} ergeben"?

Anwältin zu werden, stand wie gesagt nicht auf meinem Vorsatzzettel. Die Entscheidung, Anwältin zu werden und damit überhaupt einen juristischen Beruf auszuüben, ist während des Referendardienstes gefallen. Ich hatte das Glück, meine Anwaltsstation bei einem Anwalt zu verbringen, der seine Referendarinnen und Referendare immer sehr selbstständig hat arbeiten lassen. Das heißt, ich erfuhr relativ früh, wie der Anwaltsberuf aussieht, und zwar vom ersten Gespräch mit dem Mandaten an über die Korrespondenz und das Gerichtsverfahren bis hin zum Ende der Sache. Dabei habe ich gemerkt, dass es mir Freude macht, Menschen zu beraten, die Probleme haben, ihnen Wege aufzuzeigen, diese Probleme zu lösen und sie dabei zu begleiten. Ich fand dies damals und finde es auch heute noch eine wirklich unglaublich spannende Sache.

\section{Was interessiert Sie am Familienrecht?}

Der familienrechtliche Schwerpunkt hatte für Anwältinnen, als ich anfing, etwas geradezu Zwangsläufiges, und ich denke, so war das bei mir auch. Offensichtlich gehen Menschen in familienrechtlichen Angelegenheiten im Großen und Ganzen eher zu Anwältinnen als zu Anwälten. Mir gefällt dieses Rechtsgebiet. Ich hätte mich nie mit etwas beschäftigen können, bei dem ich keinen Kontakt zu Menschen habe und nur am Schreibtisch sitze und Papier beschreibe. Ich brauche den direkten Kontakt zu den Mandantinnen und Mandanten, zu den Menschen und ich muss auch miterleben können, wie das, was ich mache, direkte Auswirkungen auf das Leben der oder des Einzelnen hat. Das finde ich schön.

Sie waren vorher auch im Strafrecht unterwegs, da hat man den Kontakt zum Anderen ja auch?

Das hatte eher politische Gründe. Es gab, als ich mit der Berufstätigkeit anfing, sehr viele politische Aktionen, die sich am
Jutta Wagner wurde am 15. Juni 1949 in Kassel geboren und ist aufgewachsen in Dortmund, Düsseldorf, Bochum, am Bodensee und in Hamburg. Sie studierte von 1969 bis 1973 in Hamburg und Berlin Jura. Ihr erstes Examen bestand sie im August 1973. Anschließend war sie Assistentin für Ausbildungsfragen an der Freien Universität Berlin. Auch ihre Referendarzeit verbrachte sie in Berlin und legte dort im Juli 1978 das zweite Staatsexamen ab.

Seit November 1978 ist sie Rechtsanwältin. Die Jahre von 1989 bis 1991 verbrachte sie in Brüssel. Seit 1996 ist sie Notarin und seit 1997 Fachanwältin für Familienrecht in Berlin. Jutta Wagner beschäftigt sich in ihrer Kanzlei mit Fällen aus dem Familien- und Erbrecht sowie mit Notarangelegenheiten.

Von Dezember 1993 bis 2005 war sie ehrenamtliche Vorsitzende Richterin am Anwaltsgericht. Von 1985 bis 1989 war sie Mitglied im Vorstand der Rechtsanwaltskammer Berlin.

Jutta Wagner ist seit 1984 djb-Mitglied. Dem Vorstand des Landesverbands Berlin gehört sie seit 1997 an. Von 1999 bis 2004 war sie dessen Vorsitzende.

Von September 2005 bis September 2011 war sie Präsidentin des djb.

Rande der Illegalität befanden oder auch illegal waren - also diese ganze Hausbesetzerszene, die Demonstrationen und natürlich auch die Aktivitäten der dritten Generation der RAF. Aufgrund meiner eigenen politischen Aktivitäten in den 70erJahren hatte ich Kontakte und genoss ein gewisses Vertrauen. Daraus haben sich dann diese Strafverteidigungen entwickelt. Doch irgendwann musste ich einsehen, dass Familienrecht und Strafverteidigung gleichzeitig nicht ging. Es sind doch sehr unterschiedliche Betätigungsfelder und man arbeitet ganz anders. Der Spagat, wenn man das beides machen will, ist eigentlich nicht zu schaffen. Da ich bei wirklichen Kriminellen, bei deren Verteidigung man auch viel Geld verdient, nicht so richtig gut ankam, sondern mehr bei den Überzeugungstätern, habe ich mich für das Familienrecht entschieden.

\section{Hatte es politische Gründe, warum Sie von Hamburg nach Berlin gegangen sind?}

Nein, das hatte ganz persönliche Gründe. Mein damaliger Freund wollte unbedingt aus Hamburg weg, um sich von seinem Elternhaus zu lösen. Berlin war die einzige Stadt in Deutschland, die für uns in Frage kam, aber nicht aus politischen Gründen, sondern weil Berlin aus unserer damaligen Sicht jedenfalls die einzige wirkliche Großstadt in Deutschland war. Dort war kulturell sehr viel los und das hat uns einfach angezogen. 
Wie haben Sie die 70er-Jahre in West- und auch Ostberlin erlebt?

Ich fand Berlin damals wie - wenn auch anders - heute eine unglaublich spannende Stadt. Berlin war in so einem ständigen Widerspruch zwischen dem Traditionellen - die 20er-, vor allem auch die 30er-Jahre und die Nachkriegszeit waren noch unglaublich präsent. Das konnte man auf jeder Straße, in vielen Cafés und Gaststätten, in vielen Geschäften erleben. Es gab eine Welt, die so in Westdeutschland einfach nicht
Was empfehlen Sie jungen Juristinnen, speziell Anwältinnen, bei der Karriereplanung zu beachten? Ist eine Karriereplanung überhaupt nötig oder ergibt sich das dann doch irgendwie immer alles?

Karriereplanung ist mit Sicherheit nötig. Wer seine Karriere plant, hat größere Chancen, auch eine zu machen. Karriereplanung sollte eigentlich schon im Studium beginnen. Es schadet überhaupt nicht, wenn man sich relativ früh versucht zu entscheiden, welches Rechtsgebiet einen besonders

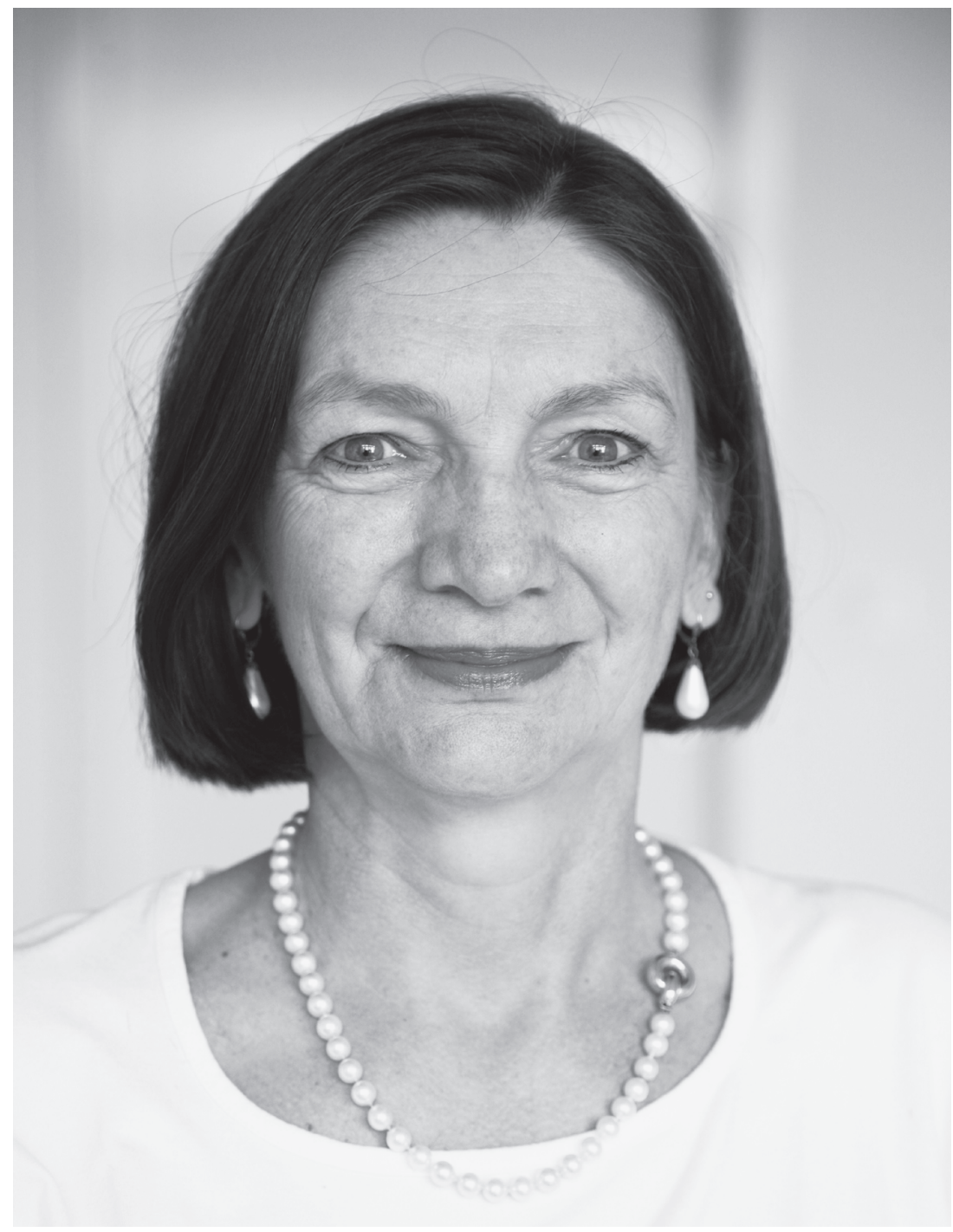

mehr vorhanden war, die schon der Modernisierung weichen musste. Andererseits gab es diesen unglaublichen Aufbruchsgeist unter den Intellektuellen, den Künstlerinn/en und den Student/inn/en, der durch die 68er eingesetzt hatte. Es war eine lebendige und spannende Zeit. Wir haben geglaubt, wir könnten die Welt verändern.

Der Ostteil Berlins war damals längst nicht so grau und deprimierend wie Ende der 80er-Jahre, es schien noch viel mehr möglich - auch dort. interessiert. Dann kann man relativ früh damit anfangen, spezielle Kenntnisse und vielleicht auch spezielle Beziehungen aufzubauen. Schon die Wahl der Stationen, die man im Referendariat absolvieren muss, kann einem bei der späteren Karriere behilflich sein. Es kommt zum Beispiel relativ häufig vor, dass die jungen Anwältinnen oder Anwälte, die bei uns - bei Redeker, Sellner, Dahs - eingestellt werden, schon ihren Referendardienst bei einer oder einem unserer Anwält/inn/e/n gemacht haben. Man stellt immer lieber jemanden 
ein, die/den man in der Praxis schon mal erlebt hat und mit der/dem man positive Erfahrungen gemacht hat. Es gibt andere Dinge: wenn man zum Beispiel zum Auswärtigen Amt will, kann man seine Referendarstation dort machen, wenn man bei einem Verband arbeiten will, kann man da als Referendarin schon hin. Wenn man in die Justiz will, ist es sicherlich nicht schlecht, sich dort gezielt besonders umzuschauen, wo man eventuell gern arbeiten möchte. Karriereplanung ist sicher etwas ganz Nützliches und im Vordergrund sollten besondere Kenntnisse und besondere Beziehungen stehen.

\section{Was ist Ihr größter Erfolg in Ihrer Karriere als Rechtsanwäl- tin?}

Das ist schwierig zu beantworten. Die spektakulären Verfahren, in denen man das Bundesverfassungsgericht dazu bringt, ein Gesetz für verfassungswidrig zu erklären oder Ähnliches, sind ja doch relativ selten. Man kommt auch relativ selten mit seinen Fällen zum Bundesgerichtshof. Eigentlich muss man sagen glücklicherweise, weil es für die Betroffenen besser ist, wenn sich die Dinge früher erledigen und nicht so spektakulär sind.

Es gibt immer einmal wieder Ergebnisse, die einen besonders freuen. Der größte Erfolg war vielleicht ein Misserfolg, als ich mit einem meiner Fälle doch einmal beim Bundesverfassungsgericht gelandet bin, was ja nicht vielen Anwältinnen und Anwälten gelingt, aus dem damaligen Westberlin schon gar nicht. Aber das Bundesverfassungsgericht entschied leider gegen mich bzw. die Mandanten. Meine unverheirateten Mandanten wollten damals die gemeinsame elterliche Sorge für ihr Kind. Inzwischen ist das ja kein Problem mehr.

Erfolge, die mich besonders freuen, sind eigentlich immer Fälle, die mit Kindern zusammenhängen. Es bewegt mich besonders, wenn ich einen Beitrag dazu leisten kann, dass deren Kindheit und Jugend durch Regelungen über das Sorgerecht, über den Aufenthalt und über den Umgang etwas weniger konfliktbeladen sind als sie es sonst vielleicht wären.

Finden Sie denn, dass die gegenwärtige rechtspolitische Entwicklung in Sachen Sorgerecht den Kindern eher nützt oder ist sie kontraproduktiv?

Bekanntermaßen bin ich eine ganz entschiedene Gegnerin einer automatischen gemeinsamen Sorge unverheirateter Eltern, weil ich überhaupt nichts davon halte, Menschen gleichberechtigt über Kinder entscheiden zu lassen, die nicht in der Lage sind, sich zu verständigen. Jedes Elternpaar, das gemeinsam sorgeberechtigt ist und damit nicht verantwortungsbewusst umgehen kann - und das kann man eben einfach nicht gesetzlich verordnen -, ist eine große Belastung für die Kinder. Man kann an die Eltern appellieren, sich vernünftig zu verständigen und gemeinsam Regelungen zu treffen, aber diese Appelle sind in Beziehungen, die wirklich gründlich misslungen sind oder vielleicht auch nie als Beziehung existiert haben, völlig sinnlos. Ausbaden müssen das die Kinder.

\section{Waren Sie die erste Juristin im Vorstand der Rechtsanwalts-} kammer Berlin?

Die erste Juristin im Vorstand der Berliner Rechtsanwaltskammer war ich nicht. Es gab in der direkten Nachkriegszeit Juristinnen, die in Vergessenheit geraten sind. In der Zeit, an die ich mich erinnere, war ich meiner Erinnerung nach die dritte. Und in der Zeit, in der ich im Kammervorstand war, war ich die einzige.

\section{Sind Sie vor dem Fall der Mauer von Berlin nach Brüssel ge- flüchtet?}

Nein, im Gegenteil, ich bin nach dem Fall der Mauer so schnell wie möglich von Brüssel nach Berlin zurückgekommen.

\section{Warum wollten Sie nicht in Brüssel bleiben? Was schätzen Sie an (West-)Berlin?}

Ich finde Berlin viel spannender als Brüssel. Und den Prozess der Vereinigung zweier Systeme, die sich vorher konfrontativ gegenüberstanden, fand ich sehr interessant. Eigentlich bin ich nach Brüssel gegangen, weil sich die Möglichkeit ergab und ich während der Studienzeit versäumt hatte, einige Zeit im Ausland zu leben. Ich hatte die Gelegenheit, das nachzuholen und habe das dann auch gemacht. Ich musste jedoch erkennen, dass ich viel deutscher bin als ich es jemals geglaubt hätte. Dazu kam der Fall der Mauer und als überzeugte Berlinerin wollte ich unbedingt dabei sein. Wir sind nach dem Mauerfall über Silvester nach Berlin gefahren. Damals habe ich beschlossen, wieder eine Wohnung in Berlin zu suchen. Ab Anfang Mai 1990 hatte ich diese kleine Wohnung und bin zunächst einmal zwischen Brüssel und Berlin gependelt: Montagmorgens nach Berlin geflogen, Donnerstagabends zurück nach Brüssel. Glücklicherweise konnte man schon kurz nach dem Mauerfall mit kleinen Maschinen von Tempelhof nach Brüssel fliegen, denn vorher hatte es keine direkten Verbindungen gegeben. Man traf dort immer wieder dieselben Leute; es war eine verschworene Gemeinschaft. Ich weiß noch, es muss auch ein Donnerstag gewesen sein, als an einem späten Nachmittag im Reichstag die Entscheidung für Berlin als Hauptstadt, als Regierungssitz gefallen ist. Der Pilot hat diese Information den Passagieren mitgeteilt und das ganze Flugzeug ist in lauten Beifall und große Freude ausgebrochen. Auch wenn man auf einem Gerichtsflur einer Kollegin, die man schon länger kannte, begegnete, fiel man sich um den Hals und brach in Tränen aus. Das war so emotional.

\section{Warum sind Sie in den djb eingetreten?}

Ich finde es trotz aller Kritik wichtig, dass Frauen organisiert ihre Rechte wahrnehmen und ausbauen. Der djb macht eine gute Rechts- und Frauenpolitik; das wollte ich unterstützen. Der Netzwerkeffekt ist auch schön, aber für mich war dies immer eher ein Nebeneffekt.

\section{Was kritisieren Sie am djb?}

Eigentlich kritisiere ich am djb nichts. Ich kritisiere höchstens die Juristinnen, die nicht im djb Mitglied sind, weil ich 
finde, dass der djb noch mehr Unterstützung gebrauchen könnte. Der djb ist ein ganz normaler Verband. Man kann hier das beanstanden, was eben bei allen Verbänden und Vereinen vorkommt: es gibt auch Intrigen und Eitelkeiten, manchmal auch Irrwege. Aber das ist völlig normal und hält sich im djb auch in übersichtlichen Grenzen. Wenn es dieses Vereinsleben mit all seinem Drum und Dran nicht gäbe, würde die Arbeit vielleicht auch gar keinen Spaß machen.

\section{Was bewog Sie 2005, als djb-Präsidentin zu kandidieren?}

Es musste ja eine machen ... Ja, warum entscheidet man sich, etwas zu tun? Weil man glaubt, es zu können und vielleicht auch glaubt, es besser zu können als andere und deshalb etwas nützen und bewegen zu können. Es hatte mir schon Spaß gemacht, den Berliner Landesverband auf einen Erfolgskurs, also nach einer Zeit der Stagnation zum Leben und zum Wachsen zu bringen. Im Grunde war die Präsidentschaft dann eigentlich ein logischer Schritt, diese Arbeit auf einer anderen Ebene fortzusetzen. Ich gehöre eher zu den Menschen, die, wenn sie irgendwas sehen, fragen: „Was kann ich tun?"

Natürlich macht es mir auch Freude, einen Verband zu repräsentieren, der so angesehen ist. Es ist einfach schön, die Anerkennung, die Achtung und den Respekt zu erleben, die dem Verband insgesamt entgegengebracht wird. Und ich rede auch gerne mit Ministerinnen, Politikerinnen, Menschen, die etwas tun können, manchmal sogar von „du zu du" und habe mindestens die Illusion, nahe bei der Macht zu sein. Sonst erlebt man das ja so nicht.

\section{Hatten Sie realistische Vorstellungen von diesem Amt? Sie wurden damit ins kalte Wasser geworfen. Und hatten Sie zwischendurch das Bedürfnis, alles hinzuwerfen oder waren diese sechs Jahre eine reine Freude?}

Ich hatte keine realistischen Vorstellungen. Ich habe nicht im Geringsten für möglich gehalten, welchen Einsatz dieses Amt erfordert, und mein Respekt gegenüber meinen Vorgängerinnen und Nachfolgerinnen ist ins Unermessliche gestiegen. Dieses Amt ergreift einen als ganze Person. Es bestimmt für die Jahre, die man es ausübt, das Leben. Das Bedürfnis, alles hinzuwerfen, hatte ich kein einziges Mal. Ich hatte eher das Bedürfnis, manchmal mehr durchzugreifen, als es möglich und gut ist ... Aber nein, so schlimm war es nie.

Was wünschen Sie dem djb? Eine lange Lebensdauer, oder haben wir schon alles erreicht?

Ich glaube, dass der djb länger leben wird als ich. Er wird noch auf eine Zeit gebraucht werden, die nicht absehbar ist. Gesellschaftliche Veränderungen brauchen viel viel mehr Zeit, als wir uns das wünschen würden, und auch manchmal als es wirklich nötig und angemessen ist. Wir sind von der Gleichstellung der Frauen noch so weit entfernt, dass ich kein Ende der Aufgaben für den djb sehe.
Ist es in Deutschland schwieriger, für die Gleichstellung der Frau einzutreten, als in anderen westeuropäischen Staaten?

Deutschland ist speziell. Sowohl die skandinavischen Staaten als auch Staaten wie Frankreich oder Großbritannien sind uns in der Beziehung voraus. Ein so konservatives Frauenbild wie es in deutschen Köpfen noch zementiert ist, kann man in Westeuropa lange suchen. Die Abqualifizierung jeder Mutter als Rabenmutter, die nicht 24 Stunden am Tag, sieben Tage in der Woche sich ausschließlich auf oft nur ein Kind konzentriert, ist ein speziell deutsches Problem.

Seit Kurzem gehören Sie der 5-köpfigen Jury für den AnneKlein-Frauenpreis der Heinrich-Böll-Stiftung an. Was ist das für ein Preis?

Der Preis wurde von Anne Klein, einer Berliner Anwaltskollegin, die dieses Jahr viel zu früh und plötzlich an Krebs gestorben ist, aus ihrem Nachlass gestiftet. Zu ihren Ehren vergibt nun die Heinrich-Böll-Stiftung den Anne-KleinFrauenpreis. Anne Klein hat als Juristin und offen lesbisch lebende Politikerin feministische Pionierarbeit geleistet. Sie war im ersten rot-grünen Berliner Senat die erste feministische Frauensenatorin. Mit dem Preis sollen Frauen geehrt werden, die sich in ganz besonderer Art und Weise für die Veränderung der Lebenssituation von Frauen in der Gesellschaft eingesetzt haben. Der Preis ist mit einem Preisgeld von 10.000 Euro verbunden, was bei Frauenpreisen relativ selten ist. Er kann insofern auch eine Unterstützung sein für Frauen, die sich frauenpolitisch stark engagieren, und deshalb denke ich, dass das eine gute Sache ist und man durch diese Preisverleihung auch etwas bewirken kann.

Wie kam es zur Einrichtung des Maria-Otto-Anwältinnenpreises des Deutschen Anwaltvereins (DAV)? Sie sind auch hier Mitglied der Preisjury?

Auch im DAV setzt sich allmählich die Erkenntnis durch, dass man sich mehr um die Anwältinnen kümmern muss und dass man mehr für Anwältinnen in Führungspositionen des DAV tun muss. Der Anwältinnenpreis ist nun das Ergebnis einer Initiative einer djb-Kollegin, Rechtsanwältin und Notarin Mechtild Düsing, die bei der American Bar Association (ABA) die Verleihung des „Margaret Brent Women Lawyers of Achievement Award“ miterlebt hat. Margaret Brent (1601-1671) war die erste Frau, die in Amerika vor einem Gericht auftrat. Die jährliche Preisverleihung an hervorragende Juristinnen ist mit 1.500 bis 2.000 Teilnehmer/inne/n die größte gesellschaftliche Veranstaltung des ABA-Jahreskongresses. Es werden Juristinnen geehrt, die anderen Juristinnen - Rechtsanwältinnen, Richterinnen und Wissenschaftlerinnen - den Weg bereitet haben. Das hat Mechtild Düsing so beeindruckt, dass sie diese Initiative erfolgreich in den DAV-Vorstand getragen hat. Maria Otto (1892-1977), die Namensgeberin des DAV-Preises, war die erste Frau, die in Deutschland als Rechtsanwältin zugelassen wurde. 
Wie wird man neben all den anderen Ämtern und Tätigkeiten zur Mitherausgeberin einer Zeitschrift, wie z.B. der FamFR - Familienrecht und Familienverfahrensrecht vom Verlag C.H.Beck?

Meine Aufgabe als Mitherausgeberin der FamFR ist es unter anderem, die Artikel vorab zu lesen und dazu meine Meinung zu äußern. Es handelt sich um eine Zeitschrift für Praktiker/innen und als Praktikerin sage ich dann dazu, das kann man gut gebrauchen, das ist irrelevant oder auch, man müsste vielleicht noch diesen oder jenen Aspekt ansprechen. Der Arbeitsaufwand hält sich also in Grenzen. Der angenehme Nebeneffekt ist, dass ich meine Pflicht, fachlich auf dem Laufenden zu bleiben, damit auch erfülle. Außerdem soll ich durch meine vielen Kontakte unter Familienrechtler/ inne/n Autorinnen oder Autoren gewinnen und allgemein werben.

Womit füllen Sie Ihre viele freie Zeit, nachdem der „Hauptjob“ als djb-Präsidentin nun weggefallen ist?

Es tut mir gut, mich nach der langen Zeit wieder intensiver um mein Büro und meine Mandantinnen und Mandanten kümmern zu können. Das macht eigentlich am meisten Freude. Die Menge an Freizeit hat sich nicht groß verändert. Also, ich kümmere mich wieder intensiver um mein Büro und meine Mandantschaft und ich denke, das tut allen Beteiligten gut. Man muss ehrlich sagen, dass es im Grunde kaum möglich ist, eine gründliche und sehr gute Arbeit als Anwältin zu leisten und gleichzeitig dieses Ehrenamt zu haben. Ich musste sehr viel improvisieren und Dinge weniger gründlich machen, als ich es gern gemacht hätte. Ich bin froh, dass ich dies wieder anders handhaben kann.

Im djbZ-Heft 4/2010 haben Sie angekündigt, dass Sie sich nun, nachdem Ihre Amtszeit als djb-Präsidentin abgelaufen ist, etwas Neues einfallen lassen werden, das Sie ehrenamtlich tun können. Haben Sie schon Pläne?

Nein, bis jetzt nicht. Das werde ich in aller Ruhe angehen. Ich werde mir vielleicht auch eher übersichtliche, auf einzelne Aktionen beschränkte Tätigkeiten suchen als mich in eine langjährige, dauerhafte Verpflichtung zu begeben.

\section{Was gibt es Unjuristisches in Ihrem Leben?}

Es tut allen meinen Freundschaften gut, dass ich jetzt wieder mehr Zeit habe, denn mein Privatleben habe ich wirklich arg vernachlässigt. Mein Mann war zum Glück immer solidarisch und hat sich nie beklagt. Außerdem kann ich wieder mehr ins Theater und ins Kino gehen, einfach mal so durch die Stadt schlendern - Luxus pur.

\section{Impressum}

\section{Schriftleitung:}

Anke Gimbal, Rechtsassessorin (V.i.S.d.P.)

Juliane Lindner

Redaktionsanschrift:

Deutscher Juristinnenbund e. V.

Anklamer Str. 38

10115 Berlin

Telefon: 030 443270-0

Telefax: 030 443270-22

E-Mail: geschaeftsstelle@djb.de

\section{Druck und Verlag:}

Nomos Verlagsgesellschaft mbH \& Co. KG

Waldseestr. 3-5

D-76530 Baden-Baden

Telefon: 07221 2104-O

Telefax: 07221 2104-27

Anzeigenverwaltung und
Anzeigenannahme:
Sales friendly, Verlagsdienstleistungen
Bettina Roos
Siegburgerstr. 123
D-53229 Bonn
Telefon: 0228 97898-o
Telefax: 0228 97898-20
E-Mail: roos@sales-friendly.de

Die Zeitschrift sowie alle in ihr enthaltenen einzelnen Beiträge und Abbildungen sind urheberrechtlich geschützt. Jede Verwertung, die nicht ausdrücklich vom Urheberrechtsgesetz zugelassen ist, bedarf der vorherigen Zustimmung des Verlages.

Namentlich gekennzeichnete Artikel müssen nicht die Meinung des Herausgebers oder der Schriftleitung wiedergeben. Unverlangt eingesendete Manuskripte - für die keine Haftung übernommen wird - gelten als Veröffentli- chungsvorschlag zu den Bedingungen des Verlags. Es werden nur unveröffentlichte Originalarbeiten angenommen. Die Verfasser erklären sich mit einer nicht sinnentstellenden redaktionellen Bearbeitung einverstanden.

\author{
Erscheinungsweise: \\ vierteljährlich \\ Bezugspreis 2011: \\ jährlich 48,-€, Einzelheft 13,-€
}

Alle Preise zzgl. Vertriebs-Direktbeordnungsgebühren inkl. MWSt.;

Bestellungen nehmen entgegen:

Der Buchhandel und der Verlag; Kündigung jeweils drei Monate zum Kalenderjahresende. Zahlungen jeweils im Voraus an: Nomos Verlagsgesellschaft, Postbank Karlsruhe, Konto 7363651 (BLZ 66010075) oder Stadtsparkasse Baden-Baden, Konto 5002266 (BLZ 66250030). 